\title{
Surface Brightness Gradients Produced by the Ring Waves of Star Formation
}

\author{
V. Korchagin ${ }^{1}$, Y.D. Mayya ${ }^{2}$, E.I. Vorobyov ${ }^{1}$ and A.K. Kembhavi ${ }^{3}$ \\ ${ }^{1}$ Institute of Physics, Stachki 194, Rostov-on-Don, Russia \\ ${ }^{2}$ Instituto Nacional de Astrofisica, Óptica y Electrónica, Apdo Postal 51 y 216, C.P. 72000, Puebla, México \\ ${ }^{3}$ Inter-University Centre for Astronomy and Astrophysics, Post Bag 4, Ganeshkhind, Pune 411007, India
}

To appear in Astrophysical Journal, March 10, 1998

\begin{abstract}
We compute surface brightness profiles of galactic disks for outwardly propagating waves of star formation with a view to investigate the stellar populations in ring galaxies. We consider two mechanisms which can create outwardly propagating star forming rings in a purely gaseous disk - a self-induced wave and a density wave. We show that the surface brightness profiles produced by both scenarios of ring formation are similar and are strongly sensitive to the velocity of the wave.

The results of our computations are compared with the observational quantities sensitive to the young and old stellar populations in the ring galaxies A0035-335 (the Cartwheel galaxy) and VIIZw466. The best fit to the observed radial $\mathrm{H} \alpha$ surface brightness distribution in the Cartwheel galaxy is obtained for a wave velocity of about 90 $\mathrm{km} / \mathrm{s}$. The red continuum brightness of the ring can be fully explained by the evolving stars present in the trailing part of the wave. However the red continuum brightness in regions internal to the ring indicates that the wave of star formation propagates in a pre-existing stellar disk in the Cartwheel. The $\mathrm{H} \alpha$ and $K$-band surface brightness profiles in VIIZw466 match the values expected from stellar populations produced by a wave of star formation propagating in a purely gaseous disk very well. We conclude that VII Zw466 is probably experiencing the first event of star formation in the disk.
\end{abstract}

Subject headings: Galaxies: individual (Cartwheel, VII Zw466) — galaxies: interactions 


\section{Introduction}

Starburst galaxies with large scale rings of star formation offer one of the rare opportunities to understand coherent star formation over scales of tens of kpc. Lynds \& Toomre (1976) and Theys \& Spiegel (1977) developed a picture of the ring formation. They demonstrated that the outwardly propagating rings can be formed by head-on or nearly head-on collisions of two disk galaxies of comparable masses. According to this picture, rings result from the differential radial oscillations excited by the gravitational perturbation of the companion galaxy. In other words, rings denote the radial zones where the stellar orbits crowd together. The density enhancements can lead to an increased rate of star formation resulting in a ring of massive stars and HII regions. This picture remained the governing scheme in interpreting the observational properties of the ring galaxies over the last two decades. Recently, Hernquist \& Weil (1993) studied the response of gas, in addition to the underlying stars, to the passage of a companion galaxy and found that the gaseous rings lag the stellar rings. This effect becomes especially noticeable when the circular density wave has propagated a significant distance into the disk and the companion and target galaxy are of similar masses.

Observations however provide growing evidence that the starburst phenomena in ring galaxies are more complicated and cannot be explained by the kinematical models. Davies \& Morton (1982) noticed that the mass of the possible companion galaxy responsible for the ring formation in the Cartwheel is about $5-10$ percent of its mass and is insufficient for the formation of the observed large ring. Neutral hydrogen observations of the Cartwheel complex of galaxies (Higdon 1996) confirmed this conclusion. Higdon found that the masses of all the three companions of the Cartwheel galaxy are individually less than six percent of the Cartwheel's mass. In a recent N-body simulation, Athanassoula, Puerari \& Bosma (1997) addressed the question of ring formation in galactic disks by infalling low-mass companions. They found companion masses of at least $20 \%$ of the target galaxy are required to produce the welldefined rings and spokes seen in the Cartwheel. There is another independent evidence against the kinematical picture of the formation of the rings in the neutral hydrogen data of Higdon. The observations show that all the neutral hydrogen in the Cartwheel is con- centrated in the outer ring region, and that there is no $\mathrm{HI}$ gas present in the inner ring or the spokes of the Cartwheel, contrary to the predictions of the collisional models. Marston \& Appleton (1995) studied a sample of ring galaxies and found the $K$-band ring (interpreted as stellar density wave) to be interior to the ring in $\mathrm{H} \alpha$, which is just the opposite of what is expected from kinematical models (Hernquist \& Weil 1993).

Observations however provide direct evidence of the collision in the recent past for the Cartwheel galaxy and VII Zw 466. Higdon (1996), in the case of the Cartwheel, and Appleton, Charmandaris \& Struck (1996) in the case of VII Zw466, detected the neutral hydrogen plumes connecting the ring galaxies with one of their nearby satellites. As of now, there exists detailed velocity field data for a small sample of ring galaxies. These observations support the expanding nature of the ring. For example, the inferred expansion velocities are $53 \mathrm{~km} / \mathrm{s}$ in the Cartwheel (Higdon 1996), $32 \mathrm{~km} / \mathrm{s}$ in VII Zw466 (Appleton et al. 1996) and $118 \mathrm{~km} / \mathrm{s}$ in Arp 143 (Higdon, Rand \& Lord 1997). There are however a few factors which do not allow unambiguous interpretation of the observed velocity fields. The above kinematical studies of the rings assumed purely planar rotating-expanding motions. A direct collision leads to strong non-planar motions of the disk particles, and hence the inferred expansion velocities may have some component from vertical motions. Moreover a kinematical fit to the observations does not determine the expansion velocity of the ring as a whole but rather the velocities of the physical motions of gas within the ring.

Thus the observational data offer strong support to the collisional origin of the rings, but at the same time are inconsistent with the predictions of the kinematic models of ring formation. Possibly the properties of ring galaxies do not depend directly on the companion's parameters. An intruder galaxy may play only the role of a "detonator", stimulating a self-organized wave of star formation. This idea was in fact first formulated by Davies \& Morton (1982) as a solution to the low companion-mass problem in the Cartwheel.

In this paper we discuss two scenarios for the formation of the large scale rings; (1) self-induced star formation by a propagating wave and (2) star formation triggered by an advancing density wave. The first model assumes that the wave is self-organized and is similar to the "fire in the forest" models discussed by Seiden \& Gerola (1982). The second model 
describes a circular density enhancement propagating outward in the disk. This is the picture expected in the kinematical scenario. Thus in the first scenario, a burst of star formation begins in the center of a gasrich galaxy, which then propagates outwards due to the massive-star induced star formation. In the second scenario, an outwardly propagating density wave triggers star formation by compressing the gas ahead of it. The central burst of star formation in the first scenario, and the onset of the density waves in the second, might have been directly triggered by a recent encounter with a companion galaxy.

We compute the surface brightness distribution in the disk for both the above mentioned scenarios of star formation, using population synthesis models of Mayya (1995). We find that the observed $\mathrm{H} \alpha$ surface brightness profiles in ring galaxies can be explained equally well for both the scenarios of the propagating wave of star formation. The detailed shapes of the radial surface brightness distributions strongly depend on the velocity of the propagating wave. A common feature for all velocities is a sharp decrease of the surface brightness immediately behind the wave of star formation for both types of wave propagation that we have considered. In contrast, the observed red continuum surface brightness profile in the Cartwheel galaxy is flatter, suggesting the wave of star formation propagates in a pre-existing stellar disk. Higdon (1996) came to the same conclusion by studying the neutral hydrogen distribution in the Cartwheel. The observed $R$ - and $K$-band surface brightness profiles in VII Zw466 can be explained if the stellar populations in this ring galaxy are produced by a fast wave of star formation propagating in a purely gaseous disk. Together with other peculiarities of this galaxy our results indicate that VII Zw466 is a unique object experiencing the first event of star formation in the disk.

In Sec. 2, we summarize the observed radial brightness profiles of ring galaxies. The models we used to compute star formation and brightness profiles are explained in Sec. 3. Model results are compared with the observational data in Sec. 4 . In Sec. 5 we present the conclusions.

\section{Radial Surface Brightness Distributions in Ring Galaxies}

Marston \& Appleton (1995) carried out an observational study of the distributions of $\mathrm{H} \alpha, R$ - and $K$ band surface brightness of a sample of ring galaxies.
The aim of their study was to determine the distributions of the old stellar populations relative to the distribution of sites of recent star formation in these galaxies. Their Fig. 11, showing normalized radial profiles of the $K$ and $\mathrm{H} \alpha$ emissions in four ring galaxies, is reproduced here in Fig. 1. The normalized radius of unity denotes the location of the ring. The most important feature to note in these plots is that the $\mathrm{H} \alpha$ profiles peak systematically at the outer edge of the $K$-band profiles in all four galaxies. As discussed earlier, the $K$-band rings trace the location of the density wave in the kinematical models and hence should be leading the regions of current star formation as traced by $\mathrm{H} \alpha$. Thus these plots offer the most compelling evidence against the interpretation of the rings in terms of kinematical models. Fig. 1 shows another important feature of the radial surface brightness distribution in ring galaxies. The $K$-band ring is much broader than the $\mathrm{H} \alpha$ ring in all four galaxies. Also, the $K$-band surface brightnesses profile of VII Zw466 is steeper compared to that of the other three galaxies. The red continuum surface brightness profile in the Cartwheel ring galaxy (Higdon 1995) follows the behavior of these 3 ring galaxies rather than VII Zw466. Hence the "empty" ring galaxy VII Zw466 is a remarkable exception. As we shall demonstrate in this paper, its radial $K, R$ and $\mathrm{H} \alpha$ surface brightness profiles can be produced by the stellar populations born in the primordial wave of star formation, where as the star forming waves are propagating on a pre-existing disk in the Cartwheel and probably other ring galaxies. If this conclusion is confirmed by future studies, the galaxy VII Zw466 can be a unique laboratory for studying star formation and the chemical evolution in galaxies.

\section{Models and Parameters}

We invoke two mechanisms of the formation of the large scale star forming rings. In the first scenario we assume that the ring galaxies are manifestations of a self-induced wave of star formation propagating in a clumpy interstellar medium. These waves were triggered in the recent past by a head-on collision with a companion galaxy. Later on, however, the wave evolved into a self-propagating wave of star formation with the properties determined mainly by the parameters of the target galaxy. The expanding star forming region LMC4 in the Large Magellanic Cloud and the associated expanding shell of neutral hydrogen discovered by Dopita, Mathewson \& Ford (1985) is proba- 
bly a smaller scale example of the self-organized star forming process taking place in the ring galaxies. The second mechanism we invoke is the "conventional" approach where the star forming wave is associated with an outwardly propagating circular density wave.

Induced star formation can operate on different scales (Elmegreen 1992). Higdon's (1995) H $\alpha$ imaging study of the Cartwheel galaxy revealed the existence of low luminosity HII regions just beyond the outer ring. Higdon interpreted these results as the secondary HII complexes spawned by the shocks or stellar winds from the luminous star forming regions in the ring. Observational data obtained by Higdon indicate that the induced star formation in the Cartwheel belongs to the large-scale triggering type according to the classification of Elmegreen (1992). The formation of giant expanding shells caused by the activity of previous generations of massive stars is a possible mechanism for triggering star formation over large scales (Palous, Franco, \& Tenorio-Tagle 1990).

The propagation of star formation in a gaseous medium with the initial surface mass density, $M_{c}$, can be described by a system of equations for balancing mass in the star forming disk (Korchagin et al. 1995):

$$
\begin{gathered}
\frac{d M_{s}}{d t}=-D+k a M_{c}(\mathbf{x}, t-T) \int d \mathbf{x}^{\prime} f\left(\mathbf{x}-\mathbf{x}^{\prime}\right) M_{s}\left(\mathbf{x}^{\prime}, t-T\right) \\
\frac{d M_{c}}{d t}=-a M_{c}(\mathbf{x}, t) \int d \mathbf{x}^{\prime} f\left(\mathbf{x}-\mathbf{x}^{\prime}\right) M_{s}\left(\mathbf{x}^{\prime}, t\right)
\end{gathered}
$$

Equation (1) describes the rate of increase of the surface density of the stars, $M_{s}$. The first term refers to the decrease of stellar density due to the death rate $D$ and the second term describes the increase of stellar density as a result of induced star formation. The coefficient $a$ is a measure of the rate of star formation, and the coefficient $k$ of the efficiency of star formation which is about a few percent as estimated from the observational data. The function $f$ represents the nonlocal "influence" of the star complexes located at point $\mathbf{x}^{\prime}$ on the interstellar medium at point $\mathbf{x}$. We chose the function $f$ to be spherically symmetric, with $f=0$ for $\left|\mathbf{x}-\mathbf{x}^{\prime}\right|>L$ where $L$ is the characteristic "radius of influence". The following normalization equation fixes the value of $f$ for $\left|\mathbf{x}-\mathbf{x}^{\prime}\right| \leq L$;

$$
\int d \mathbf{x} f(|\mathbf{x}|)=1 .
$$

The "radius of influence", $L$, together with the characteristic time of the formation of stars, $T$, are the two parameters controlling the velocity of propagation of star formation. $L$ and $T$ are chosen to be in the range of $1 \mathrm{kpc}$ and $10^{7} \mathrm{yr}$ respectively. These values are consistent with the range of parameters of star forming supershells driven by the radiation of the massive stars (Tenorio-Tagle \& Bodenheimer 1988). The gas surface density is chosen as $\mathrm{M}_{\mathrm{c}}=1.5 \times 10^{7} \mathrm{M}_{\odot} / \mathrm{kpc}^{2}$, a typical value in the irregular galaxies (Roberts \& Haynes 1994).

Observational data and theoretical arguments are consistent with the assumption that the Initial Mass Function (IMF) is a universal power-law function which does not vary with time or location of a star forming region. Therefore we used the Salpeter IMF (slope $\alpha=2.35$ ) with mass intervals $0.5 \mathrm{M}_{\odot} \leq m_{s} \leq$ $40 \mathrm{M}_{\odot}$ in our computations.

The parameter $a$ can be estimated only roughly. Our estimate is based on the following analysis. The area of the active star formation in the Cartwheel is located approximately between 15.5 and $17.5 \mathrm{kpc}$ with a star formation rate of about $67 \times 10^{6} \mathrm{M}_{\odot} / \mathrm{Myr}$ (Higdon 1995) or a total mass of stars in the star forming ring about $3 \times 10^{8} \mathrm{M}_{\odot}$. The surface density of neutral hydrogen $\left(\mathrm{M}_{c}\right.$ neglecting gas in molecular form) in the vicinity of the outer ring varies from $4 \times 10^{6} \mathrm{M}_{\odot} / \mathrm{kpc}^{2}$ to $3 \times 10^{7} \mathrm{M}_{\odot} / \mathrm{kpc}^{2}$ (Higdon 1996) We estimate $a$ with the help of Eq. (1), assuming the efficiency of star formation $k=0.1$, and the stellar mass interval $0.5 \mathrm{M}_{\odot} \leq m_{s} \leq 40 \mathrm{M}_{\odot}$ and the Salpeter's IMF. We estimate $a \sim 1$ when $M, T$ and $L$ are expressed in units of $10^{6} \mathrm{M}_{\odot}, 10^{6} \mathrm{yr}$ and $1 \mathrm{kpc}$ respectively. We varied the value of $a$ in the range $0.4-1.5$. The value of $a$ mainly determines the peak luminosity in the ring and it does not affect the shape of the surface brightness profile.

The second mechanism of star formation we consider is associated with the outwardly propagating circular density wave. In this scenario we assume that the star formation rate is proportional to the square of the density enhancement $C(r-V t)$ propagating outwardly in a homogeneous gaseous disk. The balance of the surface density of stars can then be written as:

$$
\frac{d M_{s}}{d t}=-D+k a \tilde{C}^{2}(r-V t) .
$$

The density enhancement $\tilde{C}$ is given by the gaussian function:

$$
\tilde{C}(r-V t)=A(r) \exp \left[-\frac{(r-V t)^{2}}{l^{2}}\right] .
$$


Once the IMF is fixed, the death rate $D$ can be determined from the Eqs. (1) and (3), making use of the life time of stars. We obtain the number of stars in a given radial zone as a function of mass and time by solving the Eqs (1)-(4). The total luminosity per unit surface area is then computed as:

$$
L(t)=\sum_{m} \sum_{\tau} l(m, \tau) N(m, \tau, t),
$$

where $N(m, \tau, t)$ is the number of stars per unit surface area at time $t$ with mass $m$ and age $\tau$, and $l(m, \tau)$ is the corresponding luminosity of the star. Stellar luminosities $l(m, \tau)$ are obtained with the help of stellar evolutionary (Schaller et al. 1992) and atmospheric (Kurucz 1992) models. The computations are performed at $1 / 20$ of solar metallicity, to be consistent with the low metallic abundance of the Cartwheel galaxy (Higdon 1996). The population synthesis technique used in this work is explained in detail in Mayya (1995). The results of this code are compared with that from other existing codes by Charlot (1996). Recent updates in the code are described in Mayya (1997).

\section{Results}

\subsection{Surface Brightness Distributions in a Star Forming Wave}

We performed a set of computations for different values of the radius of influence $L$ and the delay time $T$. Fig. 2 shows the red continuum and $\mathrm{H} \alpha$ line surface brightness profiles produced by the wave of induced star formation, propagating with a velocity of $90 \mathrm{~km} / \mathrm{s}$, as a function of distance from the center of the galaxy. For comparison the observed surface brightness distribution of the Cartwheel (Higdon 1995) is also shown. Note that the observed peak fluxes in both the bands are well reproduced by the model. The steep decrease of the $\mathrm{H} \alpha$ brightness on the inner part of the ring agrees very well with that from the model. On the other hand, the observed red continuum has an excess over the model values.

In the models plotted in Fig. 2, we did not invoke any special mechanism for suppression of star formation behind the advancing wave. The sharp cut-off in star formation arises because the low mass stars lock the available gas mass for the assumed parameters of IMF, as illustrated in Fig. 3. The mass contained in low-mass stars (dotted line), intermediate-mass stars (dashed line) and high-mass stars are shown separately in this figure. In massive star forming regions, it is likely that there is an in-built mechanism for the regulation of star formation. Energy deposits from massive stars in the form of stellar winds and supernova explosions are the most likely processes which bring about the suppression of star formation. We computed a model including a mechanism for suppression. To mimic the suppression, we assumed that star formation is inhibited behind the position where the high-mass stellar density peaks (see Fig. 3). The resulting profiles are shown in Fig. 4 for the same set of parameters as for Fig. 2. For Salpeter's IMF slope we get identical results with and without invoking a mechanism for the regulation of star formation. However differences can be noticed for flatter IMF slopes. The model $\mathrm{H} \alpha$ profiles with (left) and without self-regulation for an IMF slope of 1.5 is compared with the observed $\mathrm{H} \alpha$ profile of the Cartwheel galaxy in Fig. 5. Comparison of the left and right panels demonstrates that the suppression of star formation indeed leads to a sharper decrease of the $\mathrm{H} \alpha$ surface brightness behind the wave.

Our computations show that the red continuum surface brightness profile remains qualitatively the same and does not change much with the velocity of the wave or with the particular mechanism of star formation. Such a behavior allows us to make firm conclusions about the history of star formation in the Cartwheel galaxy. The observed red surface brightness in the Cartwheel increases smoothly towards the galactic center. Fig. 6 shows the residual red continuum profile in the Cartwheel obtained by subtraction of the theoretical profile from the observed red continuum profile. Except for the radial zone at $11.6 \mathrm{Mpc}$, the residual red continuum surface brightness shows a smooth decrease away from the center, reminiscent of the red continuum surface brightness distributions observed in normal disk galaxies. This result indicates that the wave in the disk of the Cartwheel propagates on a pre-existing stellar population. Higdon (1996) studying the distribution of the neutral hydrogen in this galaxy also came to the conclusion that the Cartwheel's star forming ring is not the first event of star formation in the galaxy.

At longer wavelengths, such as the $K$-band, the brightness profiles depend significantly on the duration of the star forming process, and hence on the velocity of the star forming wave and the ring radius. Fig. 7 shows $\mathrm{H} \alpha, B$-, $R$ - and $K$-band surface bright- 
ness profiles produced by the waves of star formation propagating with velocities of $14,28,45$ and 63 $\mathrm{km} / \mathrm{s}$. As we can see, the $K$-band brightness profile has prominent secondary peaks behind the position of the wave. The "ages" of these peaks, i.e. the ages of stellar populations determined by the time for the wave propagation are approximately $1.6 \times 10^{8}$ and $3.6 \times 10^{8}$ years. The origin of secondary peaks in the $K$-band profiles is caused by the interplay of the IMF, life time of stars and by the luminosity of the stars at the red giant phase. The secondary $K$-band peaks in our computations are produced by stars with masses 3-4 $\mathrm{M}_{\odot}$ entering the red giant phase after $1.6-3.4$ $\times 10^{8} \mathrm{yr}$.

Our computations demonstrate another important feature of the surface brightness distributions produced by the ring waves of star formation. In Fig. 7 we note a spatial shift between the positions of the $\mathrm{H} \alpha$ and $K$-band emissions, with the $\mathrm{H} \alpha$ peak lying outside the $K$-band peak. The amount of shift becomes increasingly noticeable for higher velocities of the wave. Thus the shifts seen in the observational data of Marston \& Appleton (1995), reproduced as Fig. 1 here, can be understood as a natural consequence of propagating waves of star formation. The sequence, II Zw28, II Hz4, VII Zw466 and LT41, of increasing observed shifts is also a sequence of increasing velocities according to our model. Thus the rings in the $K$-band do not trace regions of density enhancements, as the kinematical models suggest, rather represent evolving stars behind the advancing wave of star formation.

In the light of our computations the distribution of surface brightnesses in the galaxy VII Zw466 is very interesting. The surface brightness in this galaxy decreases interior to the ring in all the bands. A possible explanation of the peculiar surface brightness distributions in VII Zw466 is that the star forming ring in this galaxy is a manifestation of the wave of star formation propagating in a purely gaseous disk. Our computations show that the wave velocity in the galaxy VIIZw 466 should be of the order of a few tens of $\mathrm{km} / \mathrm{s}$. With this fast wave the long-living low mass stars formed interior to the ring are yet to evolve into the red giant phase. Hence the surface brightness smoothly decreases interior to the ring even in the $K$ band.

There are two measurements of the expansion velocity of the ring in VII Zw466. From optical measurements the velocity of expansion of the outer ring was found to be about $8 \mathrm{~km} / \mathrm{s}$ (Jeske 1986), and from the neutral hydrogen observations a value $32 \mathrm{~km} / \mathrm{s}$ (Appleton et al. 1996) was obtained. The latter value is likely to be more reliable due to the two-dimensional nature of the fit to the velocity fields and the high velocity resolution. The observed velocity of $32 \mathrm{~km} / \mathrm{s}$ is still a low value compared to the predictions of the wave model. The observed expansion velocity of the ring of the Cartwheel galaxy $(53 \mathrm{~km} / \mathrm{s})$ is also lower than that for our best fit models $(90 \mathrm{~km} / \mathrm{s})$. This discrepancy may be due to the fact that the velocities determined from observations are velocities of the physical motions of gas, which can only be lower than the velocity of the propagating wave itself.

The surface brightness profiles produced by densitywave induced star formation is plotted in Fig. 8. These profiles are in good quantitative agreement with the brightness distributions produced by the wave of self-induced star formation with the same velocity (Fig. 7). Thus the observed rings in the optical and near infrared bands do not represent the position of the density wave as has often been interpreted in the kinematical model. Instead, the stellar rings represent the locations where the starburst is presently in its red supergiant phase. Hence our models predict strong spectral features of red supergiant stars (Mayya 1997) at the location of the near infrared rings. Detection of such features will be a conclusive test of our models.

\section{Summary}

In this paper we have studied optical and near infrared surface brightness profiles in disk galaxies resulting from outwardly propagating waves of star formation. Our results show that the $K$ - and $R$-band emission profiles in ring galaxies can be used as a powerful tool to determine the parameters of the star forming waves. We compared the results of our model computations with the optical properties of the ring galaxies with known surface brightness distributions. Our specific results can be summarized as follows.

1. The best fit to the observed $\mathrm{H} \alpha$ and red continuum surface brightness distributions in the Cartwheel galaxy is obtained when a wave velocity of about $90 \mathrm{~km} / \mathrm{s}$ is chosen. The smooth increase of the red continuum surface brightness distribution in the Cartwheel towards the galactic center indicates however that the star formation in the advancing wave in the disk of this galaxy is not the first event of star for- 
mation. The wave in the Cartwheel's disk propagates on an underlying stellar population.

2. The observed surface brightness profiles in VII Zw466 agree with a model in which a star forming wave propagates on a purely gaseous disk. We conclude that VII Zw466 is a good candidate of a galaxy experiencing the first event of star formation in its disk.

3. The observed $\mathrm{H} \alpha$ brightness profile peaks at an outer radii compared to the $K$-band brightness profile in most of the ring galaxies. We find that this displacement is a natural consequence of an induced star formation propagating at wave velocities greater than $40 \mathrm{~km} / \mathrm{s}$.

4. The brightness profiles in galactic disks produced by a self-induced propagating star formation cannot be differentiated from the star formation triggered by an advancing density wave. The brightness profiles are sensitive to the velocity of the star forming wave.

We thank Dr. Marston for providing the postscript file of their (MA95) Fig. 11, which is reproduced as Fig. 1 here. VK would like to acknowledge Prof. K. Tomita for hospitality and Yukawa Institute for Theoretical Physics for providing financial support during the developing phases of this project.

\section{REFERENCES}

Appleton, P.N., Charmandaris, V., \& Struck, C. 1996, ApJ, 468, 532

Athanassoula, E., Puerari, I., \& Bosma, A. 1997, MNRAS, 286, 284

Charlot, S. 1996, in ASP Conf. Ser. 98, From Stars to Galaxies, Ed. C. Leitherer, U. Fritze-v. Alvensleben, \& J. Huchra (San Francisco: ASP), 275

Davies, R.L., \& Morton, D.C. 1982, MNRAS, 201, $69 \mathrm{P}$

Dopita, M.A., Mathewson, D.S. \& Ford, V.L. 1985, ApJ, 297, 599

Elmegreen, B.G. 1992, in Star Formation in Stellar Systems, ed. G. Tenorio-Tagle, M. Prieto, \& F. Sánchez (Cambridge: Cambridge University Press), 383

Hernquist, L., \& Weil, M.L. 1993, MNRAS, 261, 804
Higdon, J.L. 1995, ApJ, 455, 524

$$
\text { 1996, ApJ, 467, } 241
$$

Higdon, J.L., Rand, R.J., \& Lord, S.D. 1997, astro$\mathrm{ph} / 9709134$

Jeske, N. 1986, Ph.D. Thesis, Univ. California, Berkeley

Korchagin, V., Kembhavi, A.K., Mayya, Y.D., \& Prabhu, T.P. 1995, ApJ, 446, 574

Lynds, R., \& Toomre, A. 1976, ApJ, 209, 382

Kurucz, R.L. 1992, IAU Symp. 149, Stellar Populations of Galaxies, Ed B. Barbuy \& A. Renzini (New York: Kluwer), 225

Marston, A.P., \& Appleton, P.N. 1995, AJ, 109, 1002

Mayya, Y.D. 1995, AJ, 109, 2503

Mayya, Y.D. 1997, ApJL, 482, L149

Palous, J., Franco, J., \& Tenorio-Tagle, G. 1990, A\&A, 227, 175

Roberts, M.S., \& Haynes, M.P. 1994, ARA\&A, 32, 115

Schaller, G., Schaerer, D., Meynet, G., \& Maeder, A. 1992, A\&AS, 96, 269

Seiden, P.E., \& Gerola, H. 1982, Fundam. Cosm. Phys., 7, 241

Tenorio-Tagle,G., \& Bodenheimer, P. 1988, ARA\&A, 26,146

Theys, J.C., \& Spiegel, E.A. 1977, ApJ, 212, 616

This 2-column preprint was prepared with the AAS LATEX macros $\mathrm{v} 4.0$. 

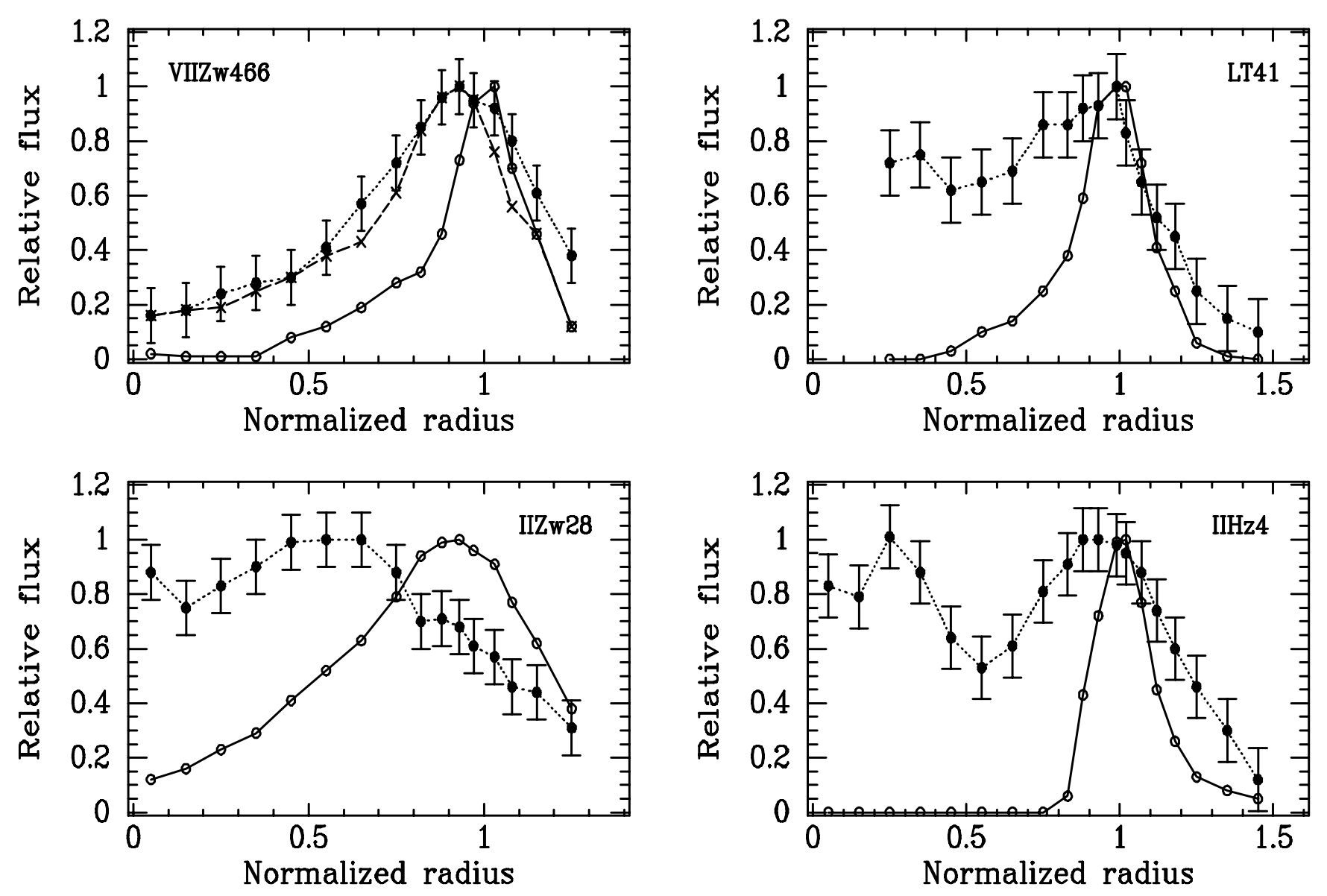

Fig. 1.- Normalized radial intensity profiles in $K$ (solid dot) and $\mathrm{H} \alpha$ (open circle) bands for four ring galaxies VII Zw466, LT41, II Zw28 and II Hz4. The symbol "X" joined by dashed line for VII Zw466 represents the $R$-band profile. Note that the $K$-band profile is broader than the $\mathrm{H} \alpha$ profile and peaks interior to that at $\mathrm{H} \alpha$. This figure is a reproduction of Fig. 11 of Marston \& Appleton (1995). 

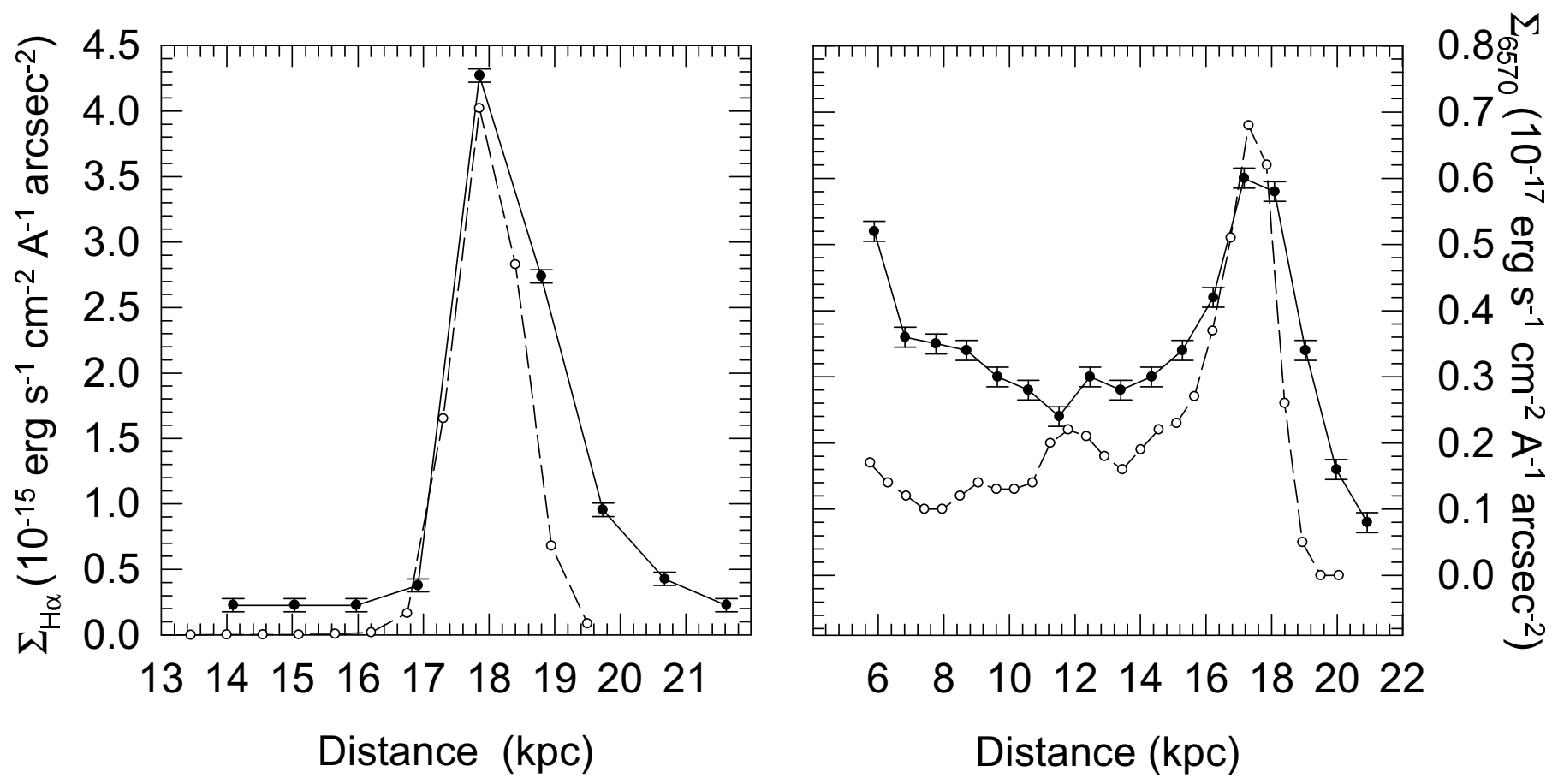

Fig. 2.- Azimuthally averaged radial surface brightness profiles in $\mathrm{H} \alpha$ (left) and red continuum (right) for the Cartwheel galaxy. Distances are measured from the center of the Cartwheel. The dashed lines with open circles correspond to the theoretical profiles with an IMF slope $\alpha=2.35$ and without self-regulation of star formation. The solid lines with filled circles represent observational profiles of Higdon (1995). Parameters for the plotted model are: $a=0.75, L=2 \mathrm{kpc}$ and $T=1.8 \times 10^{7} \mathrm{yr}$. 


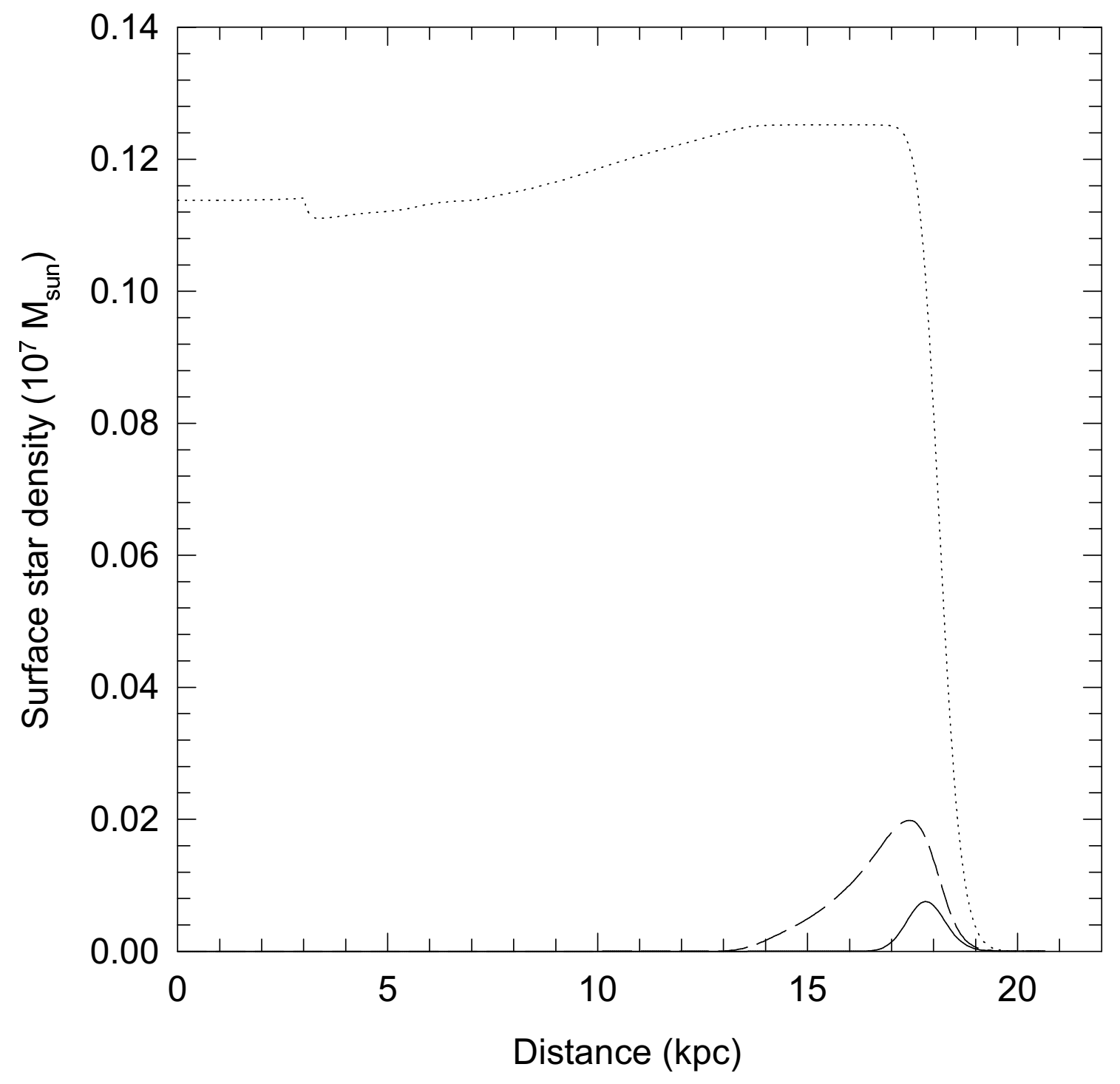

Fig. 3.- Surface star density distribution in three mass bins at the time when the wave has propagated to $17 \mathrm{kpc}$ are presented. The dotted line, dashed line and solid line represent surface density of stars in the mass range $0.5-7 \mathrm{M}_{\odot}, 7-20 \mathrm{M}_{\odot}$ and $20-40 \mathrm{M}_{\odot}$ respectively. 

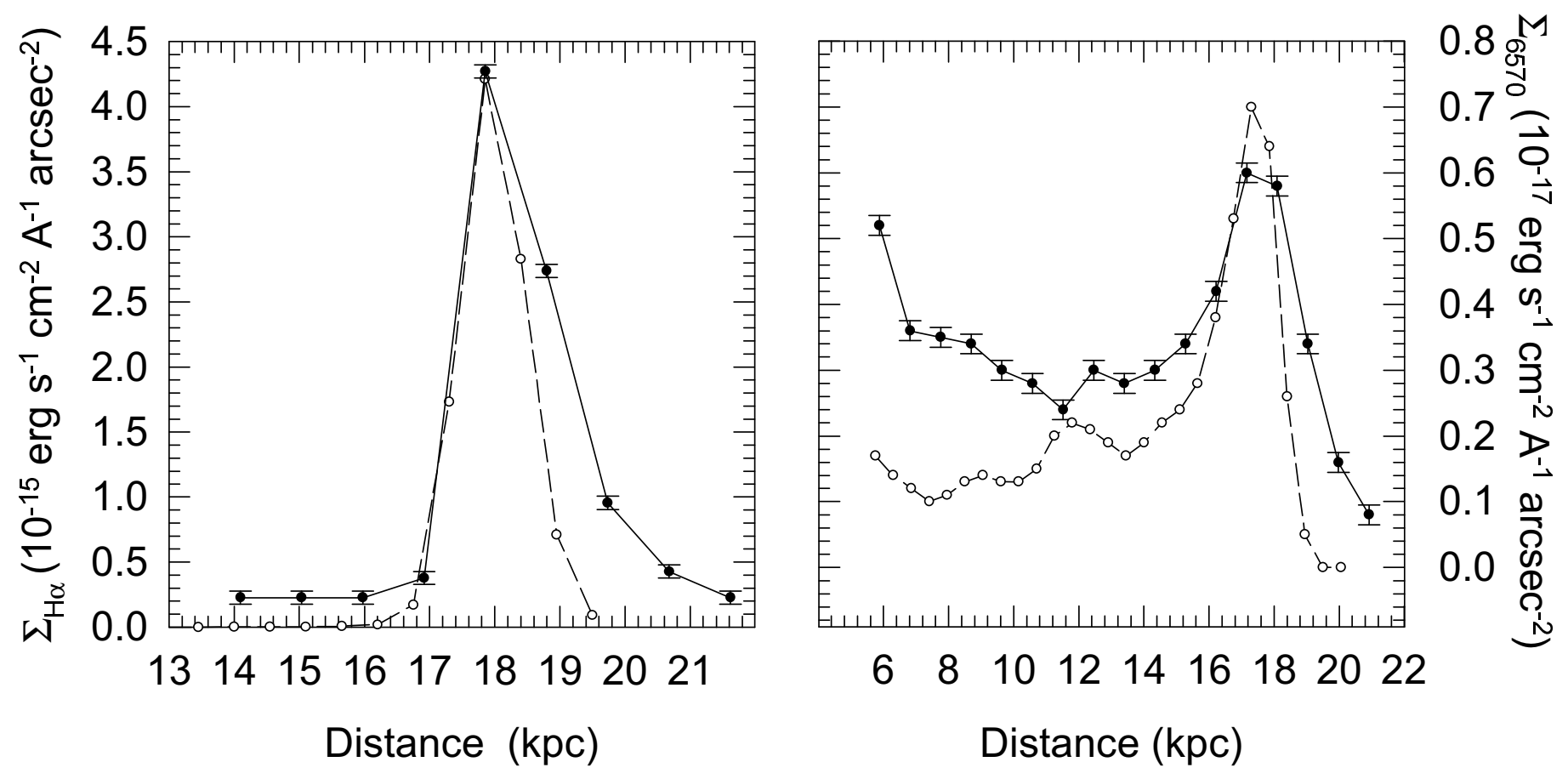

Fig. 4.- The same as Fig. 2, but with self-regulation of star formation. 

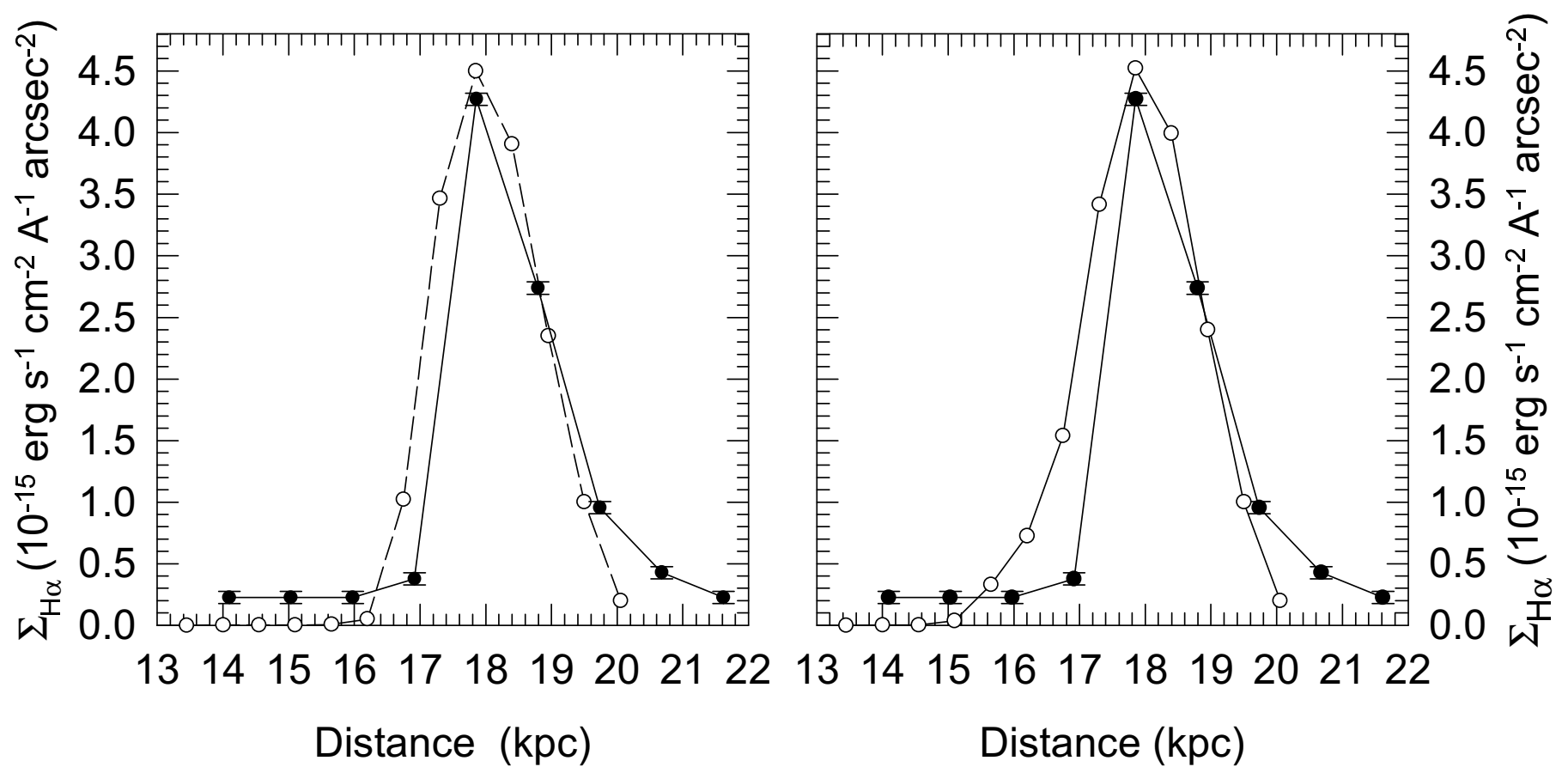

Fig. 5.- Model surface brightness profiles in $\mathrm{H} \alpha$ with (left plot) and without self-regulation of star formation are compared with the observed data of the Cartwheel (filled circle). A flat IMF with a slope $\alpha=1.5$ is used. 


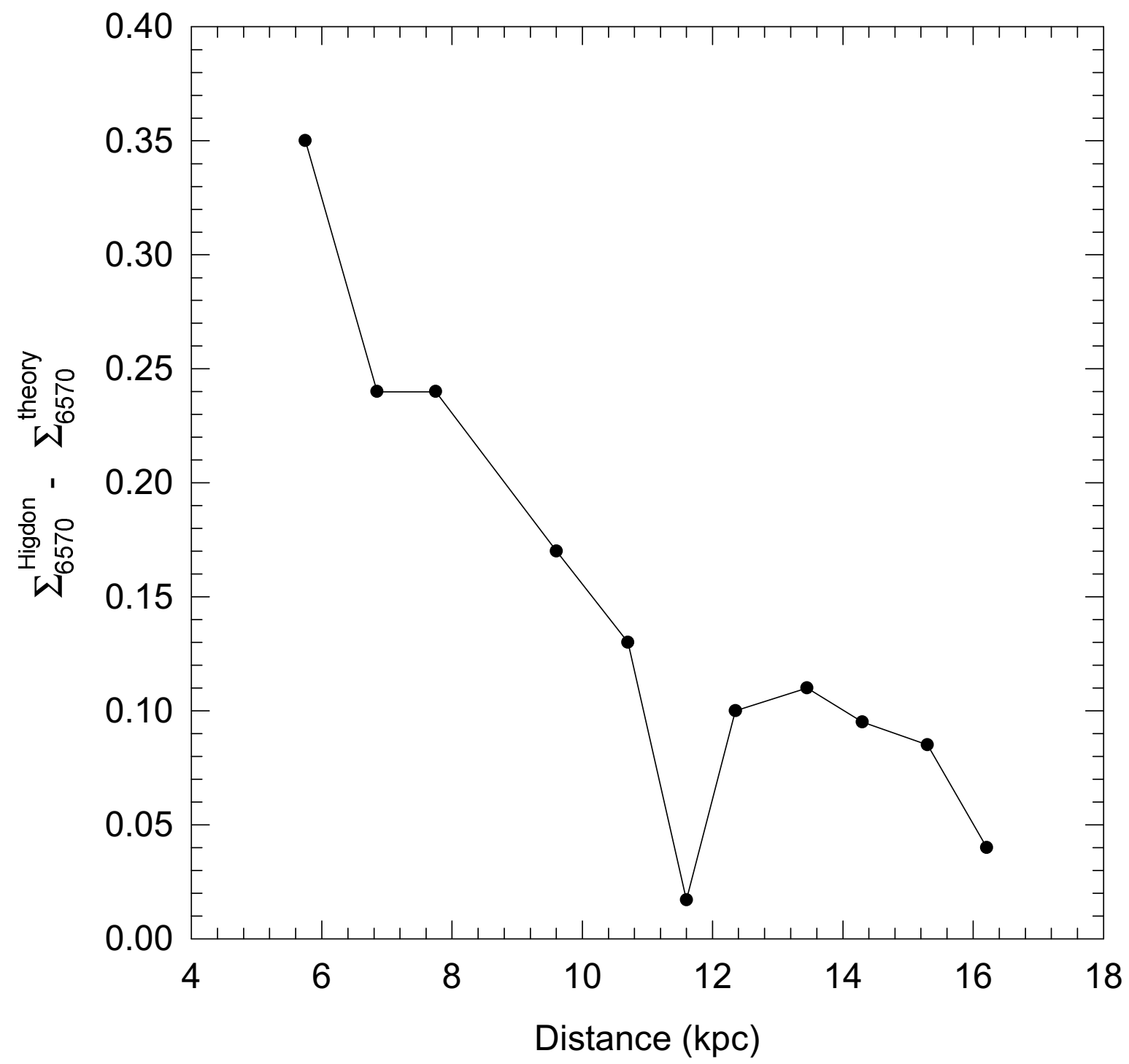

Fig. 6.- Residual profiles obtained by subtracting the model-computed red continuum surface brightness profile of Fig. 4, from the observational red continuum surface brightness profile for the Cartwheel are plotted. A smoothly decreasing residual brightness profile indicates the presence of an underlying stellar disk in the Cartwheel. 
Normalized radius $\left(\mathrm{R} / \mathrm{R}_{\mathrm{H} \alpha}\right)$

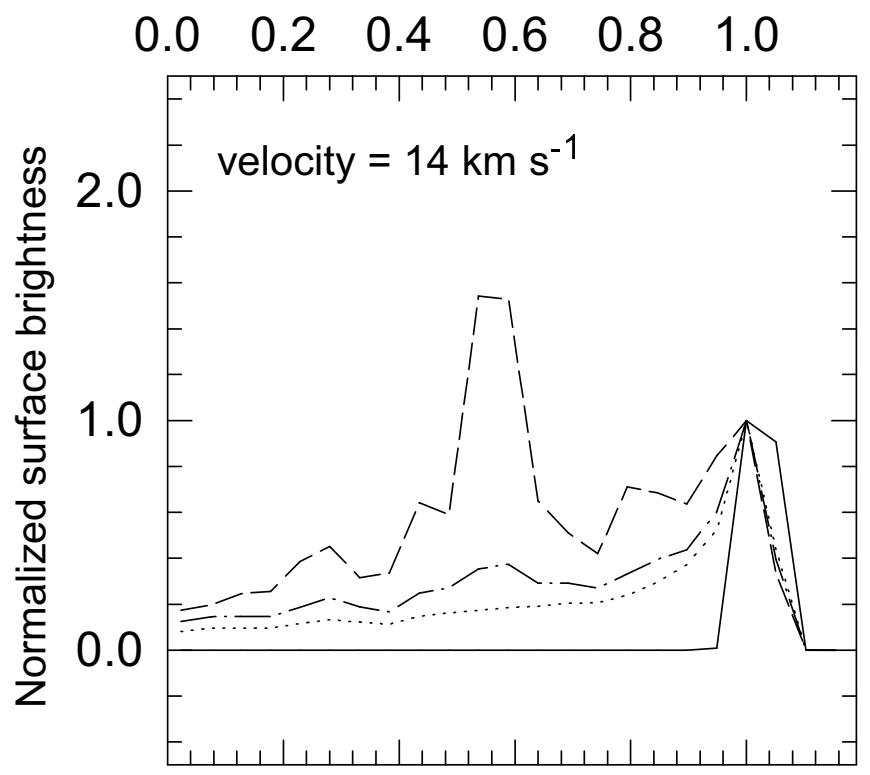

Normalized radius $\left(\mathrm{R} / \mathrm{R}_{\mathrm{H} \alpha}\right)$
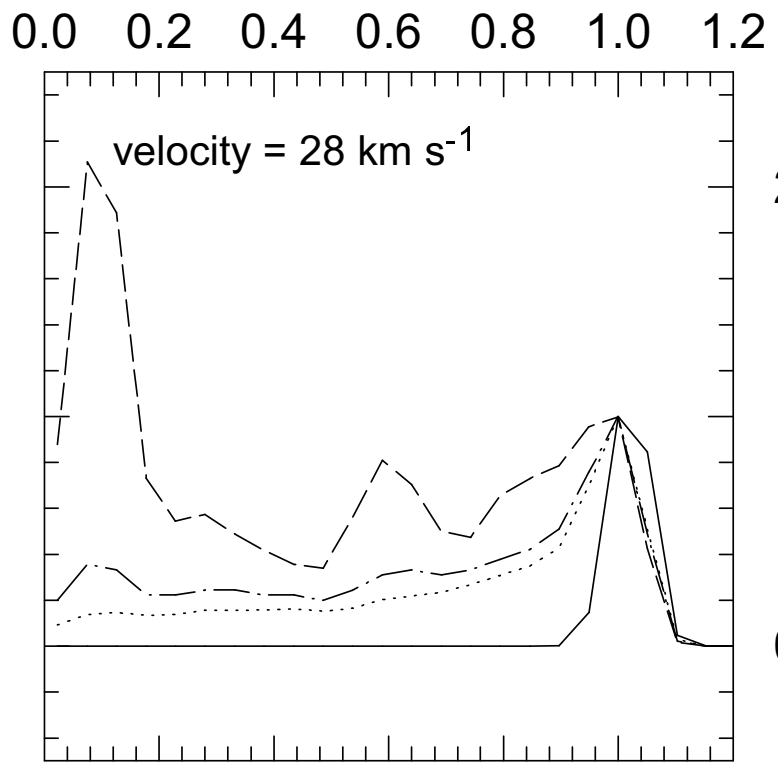

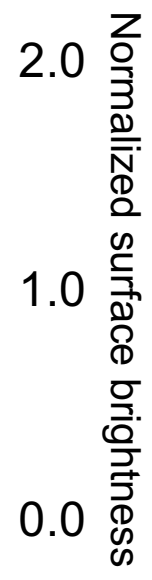

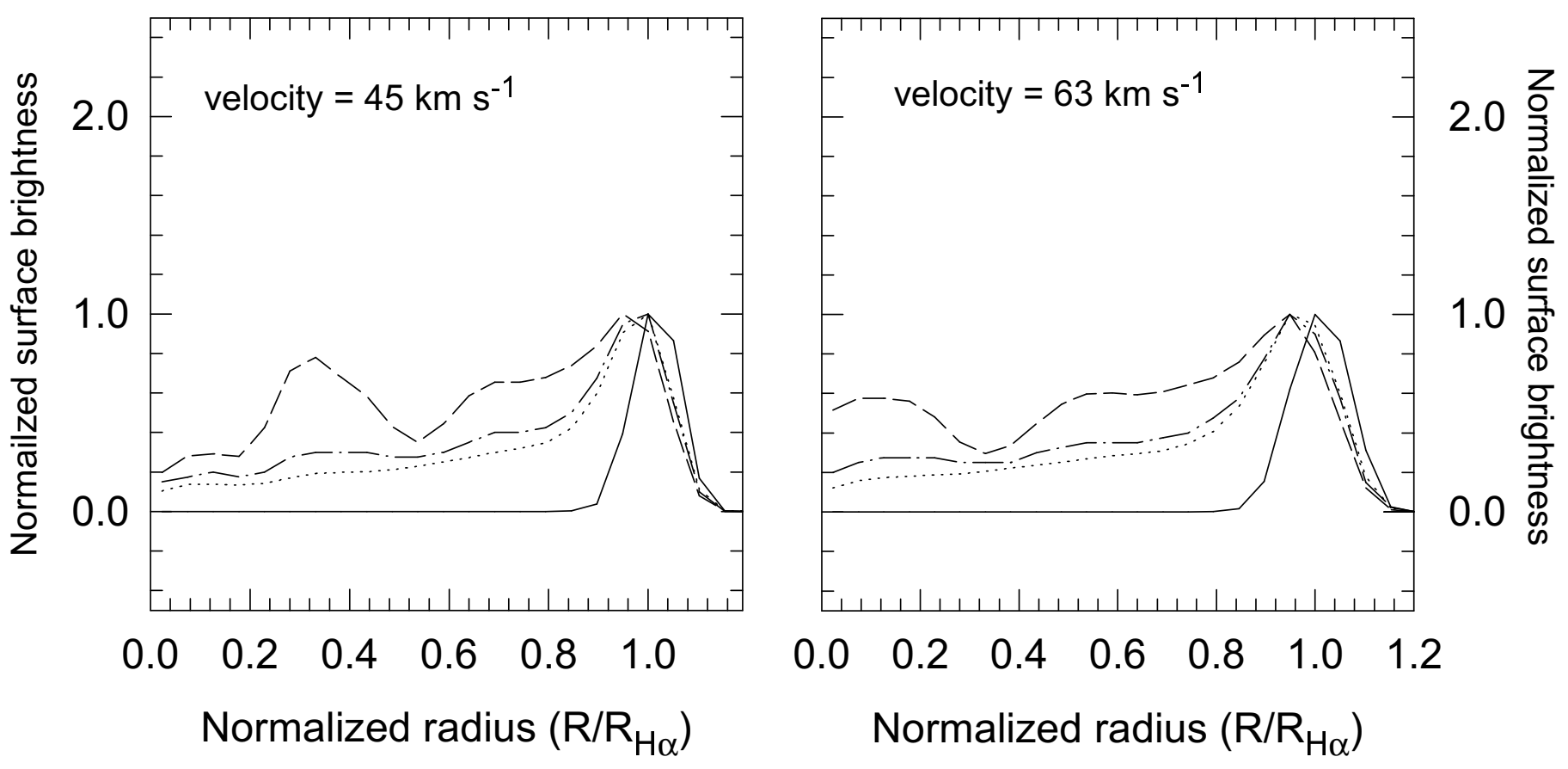

Fig. 7.- Normalized surface brightness profiles in $\mathrm{H} \alpha$ (solid line), B (dotted line), $\mathrm{R}$ (dot-dashed lines) and $\mathrm{K}$ (dashed line) bands produced by self-induced star forming waves of velocities 14, 28, 45, and $63 \mathrm{~km} / \mathrm{s}$ are shown. $R_{\mathrm{H} \alpha}$ is the position of the maximum $\mathrm{H} \alpha$ brightness. Brightness is normalized to the peak value at $R / R_{\mathrm{H} \alpha} \approx 1$. 
Normalized radius $\left(\mathrm{R} / \mathrm{R}_{\mathrm{H \alpha}}\right)$
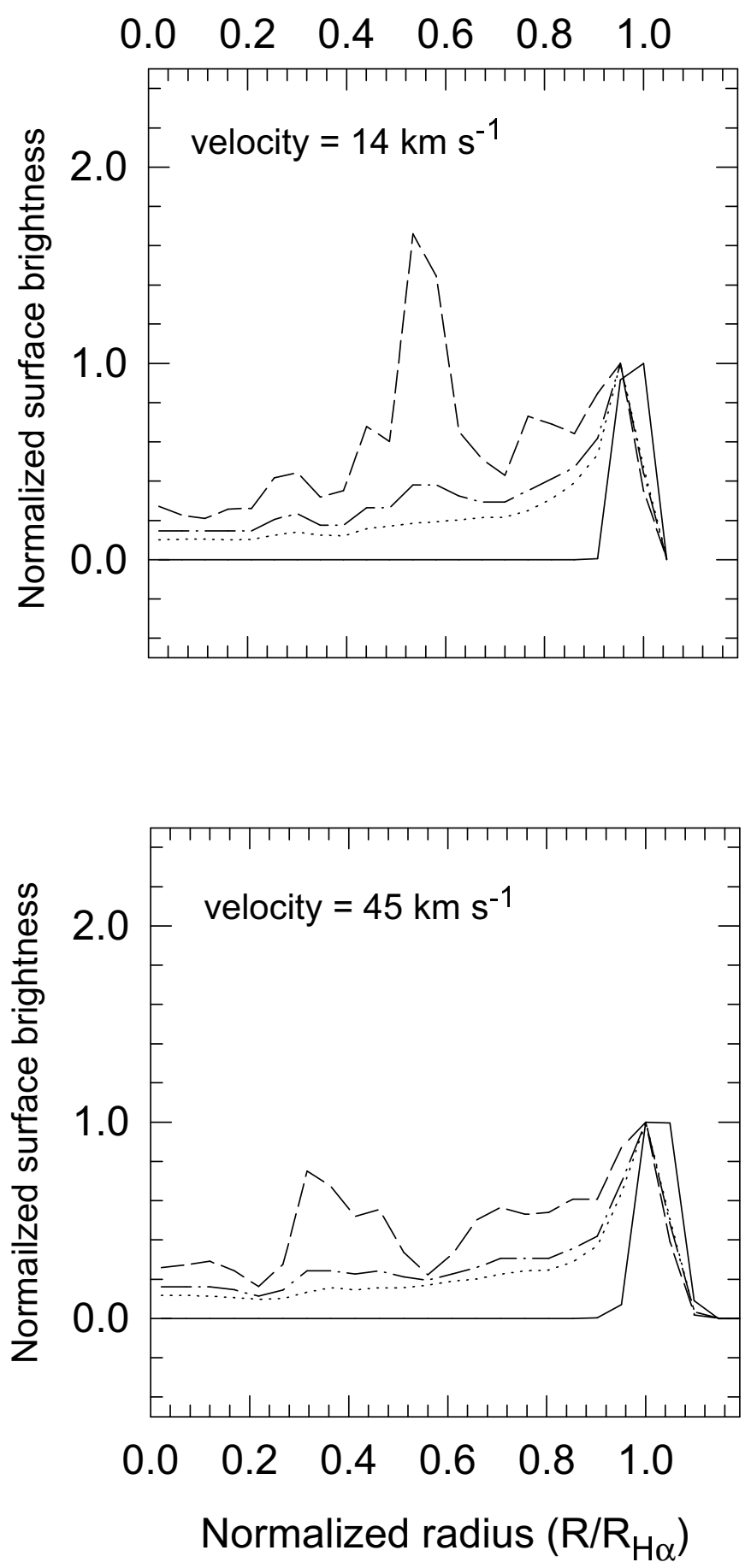

Normalized radius $\left(\mathrm{R} / \mathrm{R}_{\mathrm{H} \alpha}\right)$
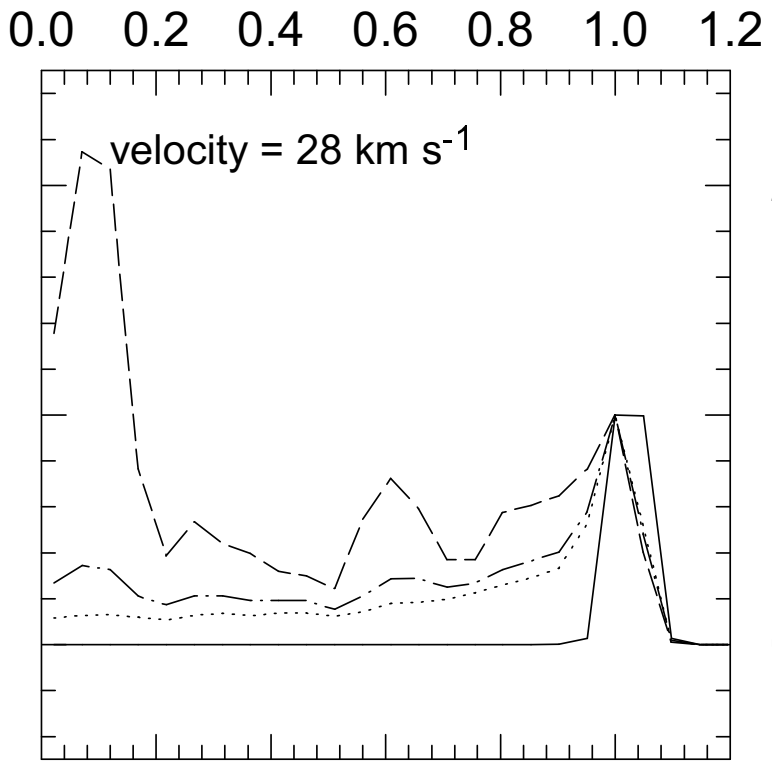

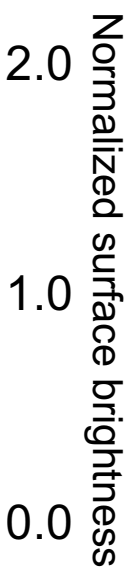

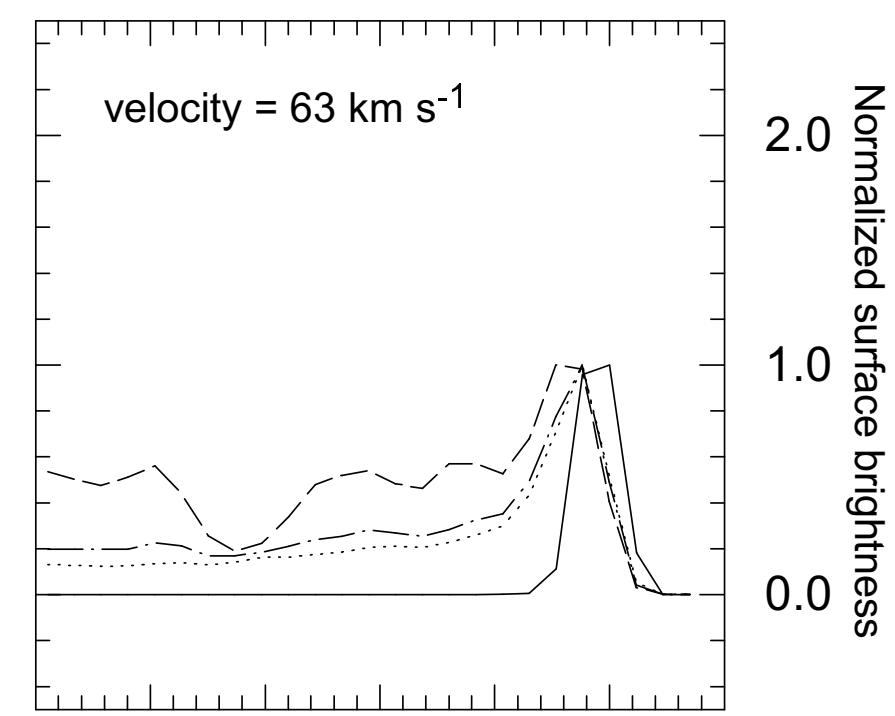

Fig. 8.- Same as Fig. 7, except that the star formation is triggered by an advancing density wave. 